\title{
ADUBAÇÃO NITROGENADA E POTÁSSICA NA PRODUTIVIDADE DA AMEIXEIRA 'REUBENNEL', NA REGIÃO DE ARAUCÁRIA - PR'
}

\author{
MARCOS ANTONIO DOLINSKI ${ }^{2}$, ANTÔNIO CARLOS VARGAS MOTTA ${ }^{3 *}$, BEATRIZ MONTE SERRAT ${ }^{3}$, \\ LOUISE LARISSA MAY DE MIO ${ }^{4}$, LINO BITTENCOURT MONTEIRO ${ }^{4}$
}

\begin{abstract}
RESUMO - A adubação é um dos fatores que podem influenciar na produção e na qualidade dos frutos, contudo existem poucas pesquisas nessa área em frutíferas no sul do Brasil para auxiliar no momento da sua recomendação. O presente trabalho teve como objetivo avaliar o efeito de doses de adubação nitrogenada e potássica para a produtividade da ameixeira (Prunus salicina), cv. 'Reubennel'. O experimento foi instalado em um pomar comercial com cinco anos, no município de Araucária-PR. O delineamento experimental utilizado foi em blocos ao acaso, distribuídos em parcelas subsubdivididas, com três repetições. Na parcela, foi aplicado

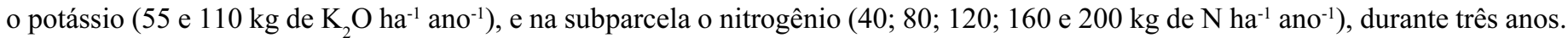
$\mathrm{O}$ fator ano foi analisado como subsubparcela. Foram avaliados a produção, o número de frutos antes do raleio e durante a colheita, e a massa e calibre dos frutos. Os resultados obtidos evidenciaram alto potencial produtivo do pomar, com uma produção média de $38,7 \mathrm{t} \mathrm{ha}^{-1}$ ano $^{-1}$, nos três anos avaliados. Contudo não foram observadas diferenças nos tratamentos e na interação entre eles para nenhuma das características avaliadas, podendo estar associado às características químicas e físicas do solo e ao efeito do manejo (poda e raleio). $\mathrm{O}$ fator ano apresentou diferença significativa para a produção, calibre e número de frutos. Independentemente dos tratamentos, a produtividade da ameixeira foi direta e inversamente proporcional ao número e tamanho dos frutos, respectivamente. As menores doses de nitrogênio e de potássio foram suficientes para obter altas produtividades durante três anos.
\end{abstract}

Termos para indexação: Ameixa, Prunus salicina, adubação, nitrogênio, potássio.

\section{NITROGEN AND POTASSIUM FERTILIZATION ON YIELD OF PLUM AT ARAUCARIA COUNTY PARANA - BRAZIL}

\begin{abstract}
Fertilization is one of the factors that can influence in the yield and quality of the fruits, however, there are few researches in that area fruit in the south of Brazil in order to guide the moment of its recommendation. The research aim was to evaluate nitrogen and potassium fertilization in the plum yield (Prunus salicina), cv. 'Reubennel'. The experiment was established in a five years old commercial orchard in Araucaria County Parana. The experiment design was a split-plot in a randomized complete block with three replications. Main plot treatments were potassium rates $\left(55\right.$ and $\left.110 \mathrm{~kg}_{\text {of K}} \mathrm{O} \mathrm{ha}^{-1} \mathrm{ano}^{-1}\right)$, and subplot treatments were the nitrogen rates $\left(40,80,120,160\right.$ and $\left.200 \mathrm{~kg}_{\text {of }} \mathrm{N} \mathrm{ha}^{-1} \mathrm{ano}^{-1}\right)$, that were applied during three years. The year factor was analyzed as split-split plot. The analyzed variables were yield, fruit number before the thinning and during harvesting, and fruit weight and caliber. The result indicated a high productive potential of the orchard used, with a mean yield of 38.7 tons $\mathrm{ha}^{-1}$ ano $^{-1}$ in the three years evaluated. However, there was no difference in the treatments or interaction effects of the treatments on any evaluated parameters which could be associated to the chemical and physics characteristics of the soil and plant management (trims and thinning). Year factor influenced yield, caliber, and fruit number. Regardless of the treatments Plum yield was direct and inverse related with fruit number and fruit size, respectively. Low $\mathrm{N}$ and $\mathrm{K}$ rates were able to obtain high yield during three years.
\end{abstract}

Index terms: Plum, Prunus salicina, fertilization, nitrogen, potassium.

\section{INTRODUÇÃO}

Em 2003, o Estado do Paraná possuía 887 hectares plantados com a cultura da ameixeira (Prunus salicina), com uma produtividade média de $10,6 \mathrm{tha}^{-1}$ (SEAB/DERAL, 2006). Todavia, atualmente, não são raros os casos de produtividades maiores que o dobro da média estadual, em pomares adequadamente manejados e sob condições climáticas favoráveis.

Dentro dos tratos culturais, a adubação é um dos fatores que pode interferir na produção, sendo que a sua resposta é dependente das características do solo, do manejo do pomar e das condições climáticas. Nitrogênio e potássio são os nutrientes exportados em maior quantidade pelos frutos de ameixeira, aproximadamente 1,61 e 1,56 kg, respectivamente, por tonelada de frutos produzida (Vitanova, 1990).

O nitrogênio, por afetar o crescimento dos ramos em tamanho e/ou número, pode interferir diretamente na produção de frutos, alterando tanto o calibre como o número de frutos produzidos (Mattos et al., 1991 e Dolinski et al., 2005). Já, o potássio, além do seu efeito direto na planta (Magnani et al., 1984 e Cummings, 1985), é também responsável pela

\footnotetext{
(Trabalho 184-06). Recebido em: 16-11-2006. Aceito para publicação em : 31-05-2007.

${ }^{2}$ Eng. Agr., Mestrando, Bolsista do Programa de Pós-graduação em Ciência do solo-mestrado. marcosagro@pop.com.br.

${ }^{3}$ Eng. Agr., Dr.(a), Professor(a) do Departamento de Solos e Engenharia Agrícola da UFPR, Rua dos Funcionários, 1540, Bairro Juvevê, Curitiba-PR - CEP 80035-050, Curitiba-PR. mottaacv@ufpr.br. *Autor para correspondência.

${ }^{4}$ Eng. Agr., Dr.(a), Professor(a) do Departamento de Fitotecnia e Fitossanitarismo da UFPR. maydemio@ufpr.br
} 
potencialização à resposta ao nitrogênio (Mengel \& Kirkby, 1987 e Marschner, 1995), sendo recomendado o aumento da dose de $\mathrm{K}$ quando é aumentada a quantidade de $\mathrm{N}$ aplicada.

Com a utilização do maior número de características para o diagnóstico da disponibilidade e do estado nutricional da planta, é possível estimar as necessidades de $\mathrm{N}$ e $\mathrm{K}$ para cada pomar. As quantidades podem variar de acordo com as características do solo, as condições das plantas e as produtividades obtidas (Vitanova, 1990).

Algumas das características de diagnóstico no momento da tomada de decisão são: a análise do solo e a foliar, e o crescimento dos ramos produtivos (Fachinello \& Herter, 2000), e ainda outros indicativos visuais, tais como: vigor da planta, emissões e diâmetro de ramos novos, coloração das folhas, tamanho e período de queda das folhas (Motta et al., 2004).

$O$ presente trabalho teve como objetivo avaliar para a cultura da ameixeira, no decorrer de três safras, o efeito de doses de nitrogênio e potássio na produtividade, no número, massa e calibre dos frutos colhidos, e, para duas safras, a produção total de frutos (antes do raleio) e a intensidade do raleio.

\section{MATERIAL E MÉTODOS}

O experimento foi instalado em maio de 2003, em um pomar comercial de ameixeira, com quatro anos de idade, da cultivar 'Reubennel', sobre porta-enxerto 'Okinawa', com espaçamento $6 \times 3 \mathrm{~m}$, conduzidas no sistema de vaso, no município de Araucária - PR, Distrito de Guajuvira. A altitude do local é $900 \mathrm{~m}$ e o clima classificado (Köppen) como Cfb (subtropical úmido), e os dados de temperatura média e precipitação acumulada mensal estão apresentadas na Tabela 1 .

O delineamento experimental utilizado foi em blocos ao acaso, com três repetições, distribuído em parcelas subsubdivididas, com cinco plantas em cada subparcela, com o potássio aplicado na parcela (55 e $110 \mathrm{~kg}$ de $\mathrm{K}_{2} \mathrm{O} \mathrm{ha}^{-1} \mathrm{ano}^{-1}$ ), na forma de cloreto de potássio, parcelado durante o início da brotação $(60 \%)$ e após o raleio $(40 \%)$. O nitrogênio foi aplicado na subparcela $\left(40 ; 80 ; 120 ; 160\right.$ e $\left.200 \mathrm{~kg} \mathrm{de} \mathrm{N} \mathrm{ha}^{-1} \mathrm{ano}^{-1}\right)$, na forma de uréia, parcelado durante o início da brotação (30\%), após o raleio $(30 \%)$ e após a colheita $(40 \%)$. O fator ano foi analisado como subsubparcela, durante três anos para a produção, o calibre e o número de frutos colhidos, e dois anos, para o número total de frutos produzidos antes do raleio e a intensidade do raleio. Todas as adubações foram realizadas manualmente na área de projeção da copa.

$\mathrm{O}$ experimento foi instalado em uma área particular, que vinha sendo conduzida com $80 \mathrm{~kg}$ de $\mathrm{Ne} 80 \mathrm{~kg}_{\text {de }} \mathrm{K}_{2} \mathrm{O} \mathrm{ha}^{-1} \mathrm{ano}^{-1}$. Com exceção dos tratamentos utilizados durante o experimento, todo o manejo adotado no pomar foi de acordo com o utilizado pelo produtor.

A análise química do solo foi realizada no primeiro ano, coletando amostras na projeção da copa e na entrelinha, para as profundidades de 0-20 e 20-40 cm, conforme metodologia descrita pela Comissão de Fertilidade do Solo (1995) (Tabela 2).

O raleio foi realizado com o objetivo de deixar os frutos no mesmo ramo, com distância mínima de $10 \mathrm{~cm}$ entre eles. A quantidade de frutos raleados foi estimada através da massa total dos frutos raleados, dividida pela massa média unitária de 100 frutos.

A produção foi avaliada durante três safras, com base na massa total dos frutos das três plantas centrais por subparcela, que foram colhidos e pesados separadamente por planta. A massa por fruto foi obtida através da média de 100 frutos por subparcela, os quais foram separados por calibre de acordo com o diâmetro: calibre 1 (d" $3,4 \mathrm{~cm})$; calibre 2 (3,5 - 4,0 cm); calibre $3(4,1-4,4 \mathrm{~cm})$; calibre 4(4,5-5,0 cm), e calibre 5 (e" $5,1 \mathrm{~cm})$.

O número de frutos colhidos foi estimado distribuindo a massa total dos frutos colhidos, proporcionalmente entre os calibres (observado para os 100 frutos), e dividindo pela massa média do calibre.

Os resultados obtidos foram analisados estatisticamente, por meio de análise de variância, e as médias foram comparadas pelo teste de Tukey, ao nível de 5\% de probabilidade.

\section{RESULTADOS E DISCUSSÃO}

Os resultados obtidos evidenciaram o alto potencial produtivo do pomar, com uma produtividade média de $38,7 \mathrm{t} \mathrm{ha}^{-1}$ ano $^{-1}$, acima da produtividade média encontrada para fruteiras de caroço (Mattos et al., 1991, Dolinski et al., 2005), e mais do triplo da média estadual para a cultura (SEAB/DERAL, 2006). Tal fato pode estar associado ao potencial produtivo da cultivar 'Reubennel', às boas condições de $\mathrm{pH}, \mathrm{V} \%, \mathrm{~K}$ e $\mathrm{P}$ do solo, e condições de formação e condução do pomar.

Para os três anos avaliados, a produtividade, o número de frutos colhidos e a massa por fruto não apresentaram diferença para os tratamentos utilizados (Tabela 3). Diferentes doses de K também não apresentaram interação com o N, e a alta concentração de K no solo explica a falta de resposta ao elemento (Tabela 2).

Contudo, nas duas menores doses de $\mathrm{N}$ utilizadas, foi observada, para o segundo e o terceiro anos, uma produtividade média de $27 \mathrm{t} \mathrm{ha}^{-1}$ ano $^{-1}$. Já, a partir das doses de $120 \mathrm{~kg}$ de $\mathrm{N} \mathrm{ha}^{-1}$ ano $^{-1}$, para os três anos avaliados, não foram verificadas produtividades médias inferiores a $31 \mathrm{tha}^{-1} \mathrm{ano}^{-1}$, sugerindo maior estabilidade na produção. Provavelmente, o período estudado não seja suficiente para gerar efeito sobre a produtividade acumulada, visto que Nosal (1990) constatou para a cultura da macieira que diferentes doses de $\mathrm{N}$ apresentaram uma diferença de $28 \%$ na produtividade acumulada de oito anos, sem diferença significativa no acumulado dos primeiros quatro anos.

Diferentemente dos tratamentos com $\mathrm{N}$ e K, o fator ano apresentou diferenças para as características citadas acima (Tabela 3). As variações entre os anos podem ocorrer por adversidades climáticas (Mattos et al., 1991, Gomes et al., 2005 e Dolinski et al., 2005), e ainda quando analisadas as plantas individualmente, se verificou uma alternância na produção por planta, tanto entre os anos como entre as plantas próximas, com uma competição e compensação na produção entre elas. Essa alternância ficou menos evidente quando se avaliou a média das três plantas centrais, uma vez que não são todas as plantas que estão em baixa ou em alta produtividade no mesmo ano, e a alternância também não é percebida pelo produtor quando se 
avalia a produção por área de um pomar.

Em 2005, quando o número de frutos colhidos foi $37 \%$ inferior ao do primeiro ano, resultou em uma redução na produtividade de $29 \%$, com um aumento de $19 \%$ na massa por fruto.

A análise de regressão entre a produção e o número de frutos colhidos por planta (Figura 1-A) mostrou que mais de $95 \%$ da oscilação observada para a produção, sem diferença significativa entre os tratamentos, pode ser explicada pelo número de frutos.

Como para a produção, a massa por fruto também foi resultado do número de frutos colhidos (Figura 1-B), sendo a produção direta e inversamente proporcional ao número e tamanho dos frutos, respectivamente. No entanto, o aumento na massa por fruto não foi suficiente para compensar a redução no número de frutos, sendo a produção resultado do número de frutos, o que foi constatado também por Mattos et al. (1991) e Dolinski et al. (2005) para a cultura do pessegueiro.

O calibre dos frutos também não apresentou diferença significativa com relação aos tratamentos utilizados (Tabela 4). Mas, para o fator ano, o calibre apresentou diferença significativa, com exceção do calibre 3, que representa quase $50 \%$ dos frutos e manteve-se inalterado, independentemente do número de frutos colhidos. A outra metade dos frutos teve a sua distribuição entre os calibres 2 e 4 dependente do número de frutos colhidos. No terceiro ano, quando o número de frutos foi $37 \%$ inferior ao primeiro ano, a porcentagem de frutos de calibre 2 passou de $43 \%$ para $15 \%$, e a de calibre 4 , de $6 \%$ para $32 \%$.

O número de frutos de calibre 2, para o fator ano, foi diretamente proporcional ao número de frutos colhidos (Figura 2-A); ao contrário, os frutos de calibre 4 foram inversamente proporcionais ao número de frutos colhidos (Figura 2-B), conforme o observado por Mattos et al. (1991), para a cultura do pessegueiro, quando a redução no número de frutos proporcionou aumento no número de frutos de primeira.

Verifica-se, no fator ano, que o tamanho do fruto a ser colhido pode ser definido em função do número de frutos que permanecer na planta, sendo o melhor tamanho de fruto para cada produtor dependente da forma de comercialização do seu produto. Deve-se levar em consideração, também, a redução na produtividade, entre os anos, observada quando se aumentou o tamanho do fruto (Tabela 3).

Como o número de frutos influencia na produção e no calibre dos frutos, conforme observado entre os anos (Tabela 3), o raleio pode ser um dos responsáveis pela falta de resposta entre os tratamentos, pois a sua intensidade define o número de frutos que permanecerão na planta.

Embora anteriormente ao raleio todas as plantas possuíssem números de frutos produzidos superiores aos números de frutos colhidos em qualquer um dos tratamentos (Tabela 5), o número de frutos que permaneceram na planta após o raleio, foi definido em função da capacidade vegetativa (tamanho e/ou número de ramos).

A relação direta observada entre o número de frutos produzidos (antes do raleio) e o número de frutos raleados foi diretamente proporcional (Figura 3-A), resultando em maior homogeneização no número de frutos colhidos. Ainda, a menor correlação entre o número de frutos produzidos (antes do raleio) e o número de frutos colhidos indica um efeito do raleio sobre o número de frutos que permaneceram na planta (Figura 3-B), o qual resultou na produtividade.

Mattos et al. (1991) concluíram para a cultura do pessegueiro que o número e o tamanho dos frutos dependem da intensidade, da uniformidade e da época de raleio, reforçado por Gomes et al. (2005), que trabalharam com a intensidade de raleio para o pessegueiro e verificaram que a maior intensidade de raleio resultou em frutos de maior calibre, reduzindo o número de frutos colhidos.

Outro fator que pode ter contribuído para a falta de resposta entre os tratamentos, é o suprimento de parte da demanda de $\mathrm{N}$ pela matéria orgânica do solo, pela presença natural de trevo-branco e pela água das chuvas. Basso \& Suzuki (1992), utilizando doses de $\mathrm{N}$ não observaram diferenças significativas para a cultura da macieira em relação à produtividade e os teores foliares, mesmo para a testemunha, e o baixo vigor e a produtividade não foram devidos apenas ao N, mas, sim, a outros fatores determinantes de vigor e produtividade.

A capacidade intrínseca da planta em manter reservas nos ramos, troncos e raízes, também pode ter contribuído para a falta de resposta, motivo pelo qual mesmo a menor dose utilizada foi suficiente para suprir a demanda de N. Tagliavini et al. (2000) verificaram que $40 \%$ do total de $\mathrm{N}$ do fruto é oriundo das reservas da planta, sendo essa contribuição mais intensa no início do desenvolvimento do fruto, mas mantendo-se em menor intensidade até a colheita.

Outra explicação para a falta de resposta entre os tratamentos pode ser o fato da homogeneização no comprimento dos ramos no momento da poda, restando apenas a diferença no número de ramos para a variação entre os tratamentos.

As características avaliadas na análise de regressão mostraram-se não-significativas para os tratamentos utilizados, sendo que a menor dose de nitrogênio e a de potássio foram suficientes para manter a produtividade e repor parte das quantidades desses elementos exportados pelos frutos. A menor dose de $\mathrm{N}$ utilizada ficou próxima da quantidade exportada pelos frutos; mesmo para a alta produtividade observada, a quantia máxima estimada de $\mathrm{N}$ exportada para as produtividades obtidas foi o equivalente a $62 \mathrm{~kg}$ de $\mathrm{N}$, valor aproximado ao observado por Vitanova (1990).

Chatzitheodorou et al. (2004) constataram que a omissão de aplicação de $\mathrm{N}$ para a cultura do pessegueiro reduziu a vida útil do pomar de forma diferenciada para as duas cultivares estudadas, sendo necessário repor ao sistema a quantidade de $\mathrm{N}$ que é exportada pelos frutos.

Somado à falta de resposta para as características avaliadas nesse trabalho, Tutida (2006) em estudo na mesma

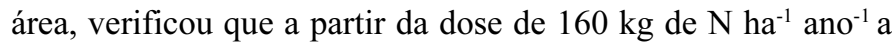
incidência de furo de bala em folhas e a de podridão-parda nos frutos foram superiores. $\mathrm{O} \mathrm{N}$ ainda interferiu na firmeza da polpa, aumentou a acidez dos frutos e retardou a sua maturação. Por outro lado, a maior dose de $\mathrm{K}$ aumentou a concentração de sólidos solúveis totais e reduziu a perda de massa por frutos após o armazenamento refrigerado, sem interação entre o $\mathrm{N}$ e o K (Tutida, 2006). 
TABELA 1 - Temperatura média $\left({ }^{\circ} \mathrm{C}\right)$ e precipitação acumulada $(\mathrm{mm})$ mensal da estação meteorológica do SIMEPAR.

\begin{tabular}{|c|c|c|c|c|c|c|c|c|c|c|c|c|c|c|c|c|c|c|c|c|c|c|c|c|}
\hline \multirow[t]{2}{*}{ Ano } & \multicolumn{2}{|c|}{ Jan } & \multicolumn{2}{|c|}{$\mathrm{Fev}$} & \multicolumn{2}{|c|}{ Mar } & \multicolumn{2}{|c|}{ Abr } & \multicolumn{2}{|c|}{ Maio } & \multicolumn{2}{|c|}{ Jun } & \multicolumn{2}{|c|}{ Jul } & \multicolumn{2}{|c|}{ Ago } & \multicolumn{2}{|c|}{ Set } & \multicolumn{2}{|c|}{ Out } & \multicolumn{2}{|c|}{ Nov } & \multicolumn{2}{|c|}{ Dez } \\
\hline & ${ }^{\circ} \mathrm{C}$ & $\mathrm{mm}$ & ${ }^{\circ} \mathrm{C}$ & $\mathrm{mm}$ & ${ }^{\circ} \mathrm{C}$ & $\mathrm{mm}$ & ${ }^{\circ} \mathrm{C}$ & $\mathrm{mm}$ & ${ }^{\circ} \mathrm{C}$ & $\mathrm{mm}$ & ${ }^{\circ} \mathrm{C}$ & $\mathrm{mm}$ & ${ }^{\circ} \mathrm{C}$ & $\mathrm{mm}$ & ${ }^{\circ} \mathrm{C}$ & $\mathrm{mm}$ & ${ }^{\circ} \mathrm{C}$ & $\mathrm{mm}$ & ${ }^{\circ} \mathrm{C}$ & $\mathrm{mm}$ & ${ }^{\circ} \mathrm{C}$ & $\mathrm{mm}$ & ${ }^{\circ} \mathrm{C}$ & $\mathrm{mm}$ \\
\hline 2002 & 21,1 & 217 & 20,3 & 120 & 22,6 & 85 & 20,5 & 123 & 17,1 & 114 & 16,5 & 40 & 13,5 & 45 & 16,9 & 90 & 15,0 & 173 & 19,9 & 122 & 19,6 & 144 & 20,9 & 154 \\
\hline 2003 & 21,2 & 148 & 23,1 & 104 & 20,5 & 165 & 18,8 & 64 & 14,8 & 19 & 16,2 & 79 & 14,7 & 121 & 13,2 & 9 & 15,6 & 130 & 17,1 & 65 & 18,7 & 119 & 19,8 & 153 \\
\hline 2004 & 19,8 & 145 & 20,2 & 60 & 19,3 & 152 & 18,9 & 87 & 13,8 & 135 & 14,3 & 58 & 13,2 & 118 & 15,0 & 11 & 18,0 & 53 & 16,5 & 152 & 18,4 & 92 & 19,4 & 128 \\
\hline 2005 & 20,4 & 165 & 20,6 & 65 & 20,7 & 73 & 19,7 & 114 & 17,1 & 105 & 16,4 & 66 & 13,9 & 91 & 16,2 & 159 & 14,0 & 195 & 17,7 & 168 & 18,5 & 78 & 19,4 & 54 \\
\hline 2006 & 22,1 & 114 & 21,6 & 144 & & & & & & & & & & & & & & & & & & & & \\
\hline
\end{tabular}

Fonte: Simepar - Sistema Meteorológico do Paraná.

TABELA 2 - Análise química do solo, da projeção da copa (PC) e na entrelinha (EL), a 0-20 e 20-40 cm de profundidade Araucária - PR, junho de 2003

\begin{tabular}{|c|c|c|c|c|c|c|c|c|c|c|c|c|c|c|c|}
\hline Local & Prof. 1 & $\begin{array}{c}\mathrm{pH} \\
\mathrm{CaCl}_{2}\end{array}$ & $\mathrm{Al}^{+3}$ & $\mathrm{H}+\mathrm{Al}^{+3}$ & $\begin{array}{l}\mathrm{Mg}^{+2} \\
--\mathrm{cmol}_{\mathrm{c}}\end{array}$ & $\begin{array}{c}\mathrm{Ca}^{+2} \\
\mathrm{dm}^{3}- \\
\end{array}$ & $\mathrm{K}^{+}$ & $\mathrm{T}$ & $\begin{array}{c}\mathrm{P}^{2} \\
\mathrm{mg} / \mathrm{dm}^{3}\end{array}$ & $\begin{array}{c}\text { M.O }^{3} \\
\%\end{array}$ & $\begin{array}{l}\mathrm{pH} \\
\text { SMP }\end{array}$ & $\begin{array}{l}\mathrm{V} \\
\%\end{array}$ & Areia & $\begin{array}{l}\text { Silte } \\
-1 \% \text { - }\end{array}$ & Argila \\
\hline $\mathrm{PC}$ & $00-20$ & 5,55 & 0 & 3,20 & 1,96 & 4,03 & 0,59 & 9,78 & 27,80 & 2,68 & 6,60 & 67 & 55 & 25 & 20 \\
\hline PC & $20-40$ & 5,40 & 0 & 3,40 & 2,01 & 3,73 & 0,30 & 9,44 & 20,43 & 2,27 & 6,50 & 64 & 53 & 28 & 19 \\
\hline EL & $00-20$ & 5,30 & 0 & 3,60 & 2,94 & 3,73 & 0,20 & 10,47 & 18,53 & 2,41 & 6,40 & 65 & 56 & 25 & 19 \\
\hline EL & $20-40$ & 5,20 & 0 & 3,60 & 3,08 & 3,68 & 0,09 & 10,45 & 9,77 & 2,00 & 6,40 & 66 & 50 & 28 & 22 \\
\hline
\end{tabular}

${ }^{1}$ Profundidade da coleta em $\mathrm{cm} .{ }^{2}$ Extrator Mehlich. ${ }^{3}$ Matéria orgânica

TABELA 3 - Produtividade, número de frutos colhidos e massa média por fruto, da ameixeira 'Reubennel', sob diferentes doses de nitrogênio e potássio (Araucária - PR)

\begin{tabular}{|c|c|c|c|c|c|c|c|c|c|c|}
\hline \multirow{2}{*}{$\begin{array}{l}\text { Tratamentos } \\
\left(\mathrm{kg} \mathrm{ha}^{-1} \mathrm{ano}^{-1}\right)\end{array}$} & \multicolumn{4}{|c|}{$\begin{array}{l}\text { Produtividade } \\
\qquad\left(\mathrm{t} \mathrm{ha}^{-1}\right)\end{array}$} & \multicolumn{3}{|c|}{$\begin{array}{l}\text { Número de frutos } \\
\text { (por planta) }\end{array}$} & \multicolumn{3}{|c|}{$\begin{array}{l}\text { Massa por fruto } \\
\quad \text { (gramas) }\end{array}$} \\
\hline & 2003 & 2004 & 2005 & $\mathrm{PA}^{4}$ & 2003 & 2004 & 2005 & 2003 & 2004 & 2005 \\
\hline $40 \mathrm{~N}^{1}$ & 49,5 & 27,4 & 38,1 & 115 & 1631 & 939 & 1127 & 48 & 62 & 60 \\
\hline $80 \mathrm{~N}$ & 37,9 & 42,3 & 27,0 & 107 & 1208 & 1335 & 813 & 55 & 56 & 69 \\
\hline $120 \mathrm{~N}$ & 45,8 & 40,1 & 31,2 & 117 & 1559 & 1296 & 922 & 53 & 59 & 65 \\
\hline $160 \mathrm{~N}$ & 42,0 & 39,0 & 31,9 & 113 & 1365 & 1247 & 905 & 56 & 60 & 67 \\
\hline $200 \mathrm{~N}$ & 55,8 & 35,5 & 36,5 & 128 & 1831 & 1077 & 1025 & 53 & 63 & 63 \\
\hline Média & 46,2 a & 36,9 b & $32,9 \mathrm{~b}$ & 116 & 1519 a & $1179 \mathrm{~b}$ & $958 \mathrm{~b}$ & $53 \mathrm{c}$ & $60 \mathrm{~b}$ & $65 \mathrm{a}$ \\
\hline $55 \mathrm{~K}^{2}$ & 47,1 & 38,8 & 30,2 & 116 & 1543 & 1242 & 885 & 54 & 59 & 66 \\
\hline $110 \mathrm{~K}$ & 45,2 & 35,0 & 35,6 & 116 & 1495 & 1115 & 1032 & 53 & 61 & 64 \\
\hline C.V. $(\%)^{3}$ & & 33,48 & & & & 32,17 & & & 9,41 & \\
\hline
\end{tabular}

${ }^{1} \mathrm{~N}$ Nitrogênio. ${ }^{2} \mathrm{~K}$ Potássio ( $\left.\mathrm{K}_{2} \mathrm{O}\right) .{ }^{3} \mathrm{C}$.V.(\%) Coeficiente de variação. ${ }^{4} \mathrm{PA}$ Produtividade acumulada.

Médias seguidas da mesma letra na linha não diferem entre si, pelo teste de Tukey, ao nível de $5 \%$ de probabilidade.

TABELA 4 - Percentual de frutos dos diversos calibres, da ameixeira 'Reubennel', sob diferentes doses de nitrogênio e potássio (Araucária - PR)

\begin{tabular}{|c|c|c|c|c|c|c|c|c|c|c|c|c|c|c|c|}
\hline \multirow{2}{*}{$\begin{array}{l}\text { Tratamentos } \\
\left(\mathrm{kg} \mathrm{ha}^{-1} \mathrm{ano}^{-1}\right)\end{array}$} & \multicolumn{3}{|c|}{1} & \multicolumn{3}{|c|}{2} & \multicolumn{3}{|c|}{3} & \multicolumn{3}{|c|}{4} & \multicolumn{3}{|c|}{5} \\
\hline & 2003 & 2004 & 2005 & 2003 & 2004 & 2005 & 2003 & 2004 & 2005 & 2003 & 2004 & 2005 & 2003 & 2004 & 2005 \\
\hline $40 \mathrm{~N}^{1}$ & 08 & 03 & 0 & 57 & 22 & 21 & 33 & 44 & 54 & 02 & 31 & 23 & 0 & 0 & 02 \\
\hline $80 \mathrm{~N}$ & 07 & 04 & 0 & 39 & 31 & 11 & 45 & 49 & 41 & 09 & 16 & 42 & 0 & 0 & 06 \\
\hline $120 \mathrm{~N}$ & 06 & 05 & 0 & 43 & 26 & 16 & 46 & 47 & 51 & 05 & 22 & 30 & 0 & 0 & 03 \\
\hline $160 \mathrm{~N}$ & 05 & 03 & 0 & 35 & 23 & 12 & 50 & 47 & 49 & 10 & 27 & 34 & 0 & 0 & 05 \\
\hline $200 \mathrm{~N}$ & 07 & 03 & 0 & 42 & 21 & 16 & 44 & 44 & 50 & 07 & 32 & 31 & 0 & 0 & 03 \\
\hline Média & $7 \mathrm{a}$ & $4 \mathrm{~b}$ & Oc & $43 \mathrm{a}$ & $25 \mathrm{~b}$ & $15 \mathrm{c}$ & $44 \mathrm{a}$ & $46 \mathrm{a}$ & $48 \mathrm{a}$ & $6 \mathrm{~b}$ & 25 a & $32 \mathrm{a}$ & $\mathbf{0} \mathbf{b}$ & $\mathbf{0 ~ b}$ & $3 \mathrm{a}$ \\
\hline $55 \mathrm{~K}^{2}$ & 08 & 03 & 00 & 43 & 27 & 16 & 41 & 48 & 46 & 8 & 22 & 34 & 0 & 0 & 04 \\
\hline $110 \mathrm{~K}$ & 06 & 04 & 00 & 43 & 23 & 16 & 47 & 44 & 51 & 4 & 29 & 30 & 0 & 0 & 03 \\
\hline C.V. $(\%)^{3}$ & & 29,35 & & & & & & 23,16 & & & & & & 37,84 & \\
\hline
\end{tabular}

${ }^{1} \mathrm{~N}$ Nitrogênio. ${ }^{2} \mathrm{~K}$ Potássio $\left(\mathrm{K}_{2} \mathrm{O}\right) .{ }^{3} \mathrm{C} . \mathrm{V} .(\%)$ Coeficiente de variação.

Médias seguidas da mesma letra na linha não diferem entre si, pelo teste de Tukey, ao nível de 5\% de probabilidade. 
TABELA 5 - Número de frutos produzidos anteriormente ao raleio (NFP), número de frutos raleados (NFR), número de frutos colhidos (NFC), porcentagem de frutos raleados em relação ao fruto total produzido (PFR), e massa média por fruto raleado em gramas (MFR), de ameixeira 'Reubennel', sob diferentes doses de nitrogênio e potássio (Araucária - PR)

\begin{tabular}{|c|c|c|c|c|c|c|c|c|c|c|}
\hline \multirow{2}{*}{$\begin{array}{l}\text { Tratamentos } \\
\left(\mathrm{kg} \mathrm{ha}^{-1} \mathrm{ano}^{-1}\right)\end{array}$} & \multicolumn{2}{|c|}{ NFP } & \multicolumn{2}{|c|}{ NFR } & \multicolumn{2}{|c|}{ NFC } & \multicolumn{2}{|c|}{ PFR } & \multicolumn{2}{|c|}{ MFR } \\
\hline & 2004 & 2005 & 2004 & 2005 & 2004 & 2005 & 2004 & 2005 & 2004 & 2005 \\
\hline $40 \mathrm{~N}^{1}$ & 1880 & 4944 & 1032 & 3697 & 848 & 1247 & 56 & 74 & 8,9 & 8,2 \\
\hline $80 \mathrm{~N}$ & 4511 & 2644 & 3085 & 1687 & 1426 & 958 & 67 & 62 & 8,1 & 9,2 \\
\hline $120 \mathrm{~N}$ & 3198 & 4170 & 2114 & 3108 & 1084 & 1062 & 58 & 62 & 8,4 & 6,9 \\
\hline $160 \mathrm{~N}$ & 3423 & 4534 & 2204 & 3298 & 1219 & 1236 & 62 & 66 & 9,1 & 8,7 \\
\hline $200 \mathrm{~N}$ & 3231 & 4381 & 2175 & 3231 & 1057 & 1150 & 62 & 73 & 9,4 & 7,8 \\
\hline Média & 3249 & 4135 & 2122 & 3004 & 1127 & 1131 & 61 & 67 & 8,8 & 8,1 \\
\hline $55 \mathrm{~K}^{2}$ & 3481 & 3926 & 2167 & 2862 & 1314 & 1064 & 58 & 67 & 8,3 & 8,9 \\
\hline $110 \mathrm{~K}$ & 3016 & 4344 & 2077 & 3146 & 940 & 1197 & 64 & 67 & 9,3 & 7,4 \\
\hline C.V. $(\%)^{3}$ & \multicolumn{2}{|c|}{49,75} & \multicolumn{2}{|c|}{$\mathbf{5 6 , 5 5}$} & \multicolumn{2}{|c|}{45,51} & \multicolumn{2}{|c|}{27,81} & \multicolumn{2}{|c|}{21,16} \\
\hline
\end{tabular}

${ }^{1} \mathrm{~N}$ Nitrogênio. ${ }^{2} \mathrm{~K}$ Potássio $\left(\mathrm{K}_{2} \mathrm{O}\right) .{ }^{3}$ C.V.(\%) Coeficiente de variação.

A.

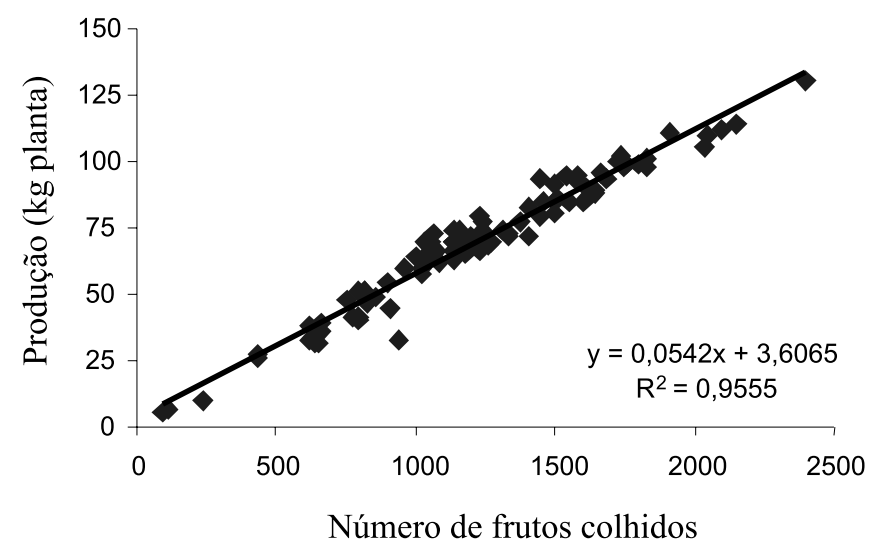

B.

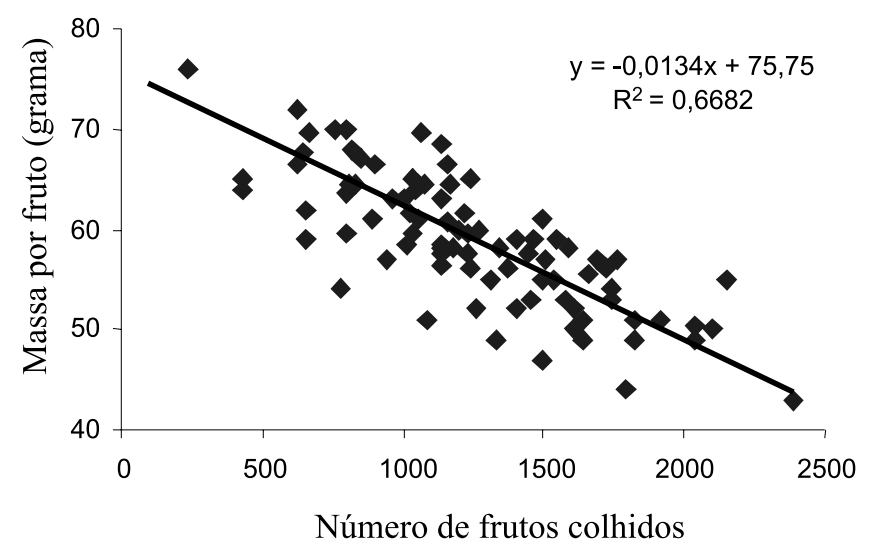

FIGURA 1 - Produção por planta $\left(\mathrm{kg} \mathrm{planta}^{-1}\right)$ (A) e massa por fruto (grama) (B), em função do número de frutos colhidos, da ameixeira 'Reubennel', sob diferentes doses de nitrogênio e potássio (Araucária - PR).

A.

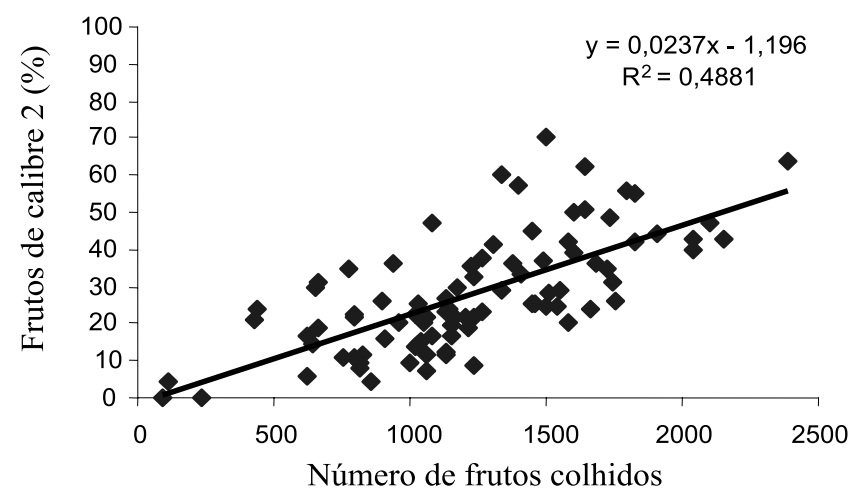

B.

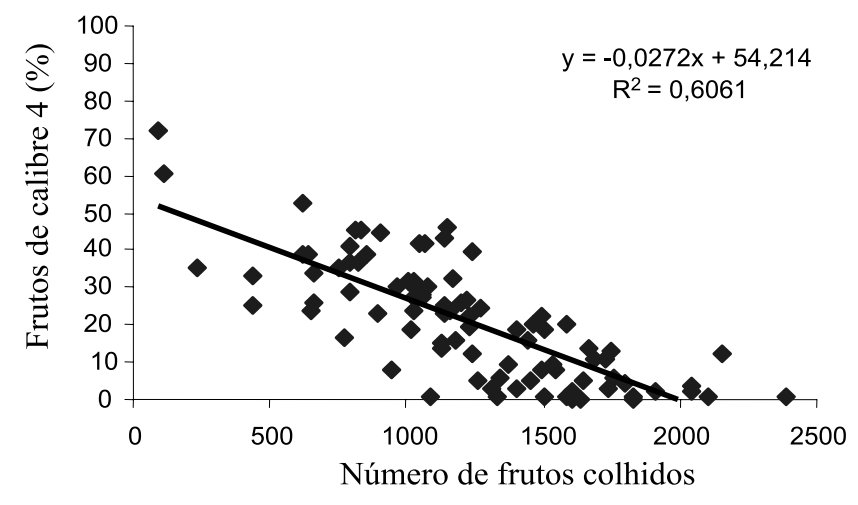

FIGURA 2 - Porcentagem de frutos de calibre 2 (\%) (A), e de calibre 4 (\%) (B), em função do número de frutos colhidos, da ameixeira 'Reubennel', sob diferentes doses de nitrogênio e potássio (Araucária - PR). 
A.

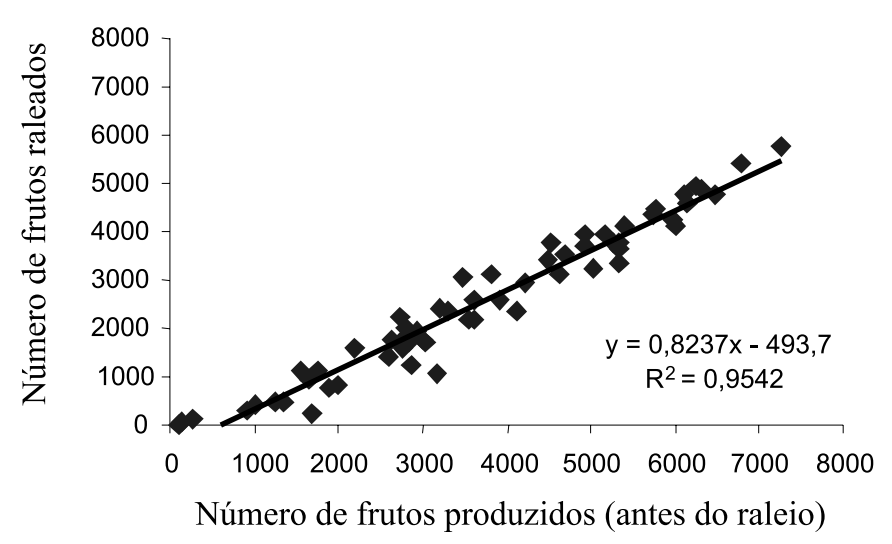

B.

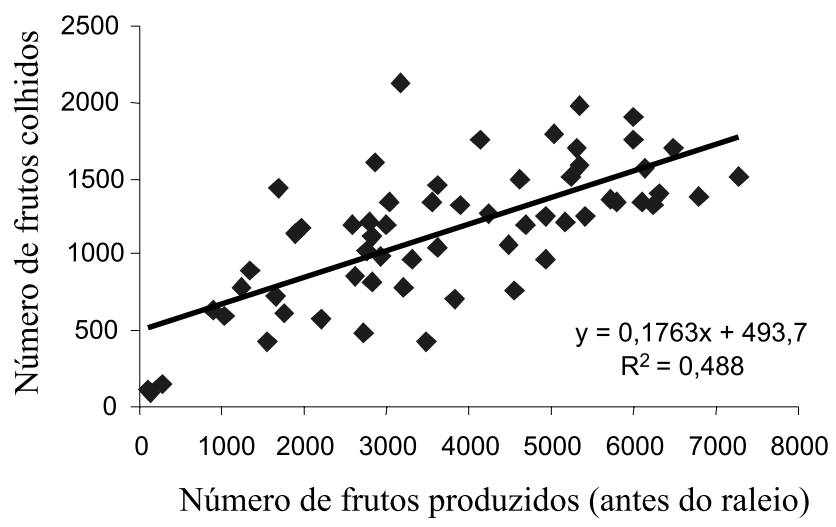

FIGURA 3 - Número de frutos raleados por planta (A) e número de frutos colhidos (B), em função do número de frutos produzidos (antes do raleio) da ameixeira 'Reubennel', sob diferentes doses de nitrogênio e potássio (Araucária - PR).

\section{CONCLUSÕES}

1. A menor dose de $\mathrm{N}$ e de $\mathrm{K}$, a partir do quinto ano de implantação do pomar é suficiente para manter a produtividade durante três anos.

2. Alterando apenas o $\mathrm{N}$ e o $\mathrm{K}$ no pomar com cinco anos de idade, por um período de três anos, não foi suficiente para promover alteração na produção.

3. A falta de resposta ao $\mathrm{N}$ e ao $\mathrm{K}$, acompanhada da alta produtividade, indica que essa é resultante de outras características e do manejo do pomar.

4. Para o fator ano, as diferenças na produção apresentaram relação direta com o número e inversa com o calibre dos frutos colhidos.

\section{AGRADECIMENTOS}

Ao Sr. Waldomiro Gayer Neto, por conceder o pomar para a instalação do experimento e por todo o apoio dado no campo para o desenvolvimento do trabalho. Ao Conselho Nacional de Apoio à Pesquisa (CNPq) e ao Ministério da Agricultura e Abastecimento (MAPA), pelo suporte financeiro.

\section{REFERÊNCIAS}

BASSO, C.; SUZUKI, A. Resposta da macieira cv. Golden Delicious à adubação nitrogenada, Revista Brasileira de Ciência do Solo, Campinas-SP, v.16, n.2, p.223-227, 1992.

CHATZITHEODOROU,I.T.; SOTIROPOULOS,T.E.; MOUHTARIDOU,G.I. Effect of nitrogen, phosphorus, potassium fertilization and manure on fruit yield and fruit quality of the peach cultivars 'Spring Time' and 'Red Haven'. Agronomy Research, v.2, n.2, p.135-143, 2004.

COMISSÃO DE FERTILIDADDE DO SOLO RS/SC. Recomendação de adubação e de calagem para os estados do RS e de SC. 3.ed. Passo Fundo-RS, Sociedade Brasileira de Ciência do Solo, 1995. 224p.
CUMMINGS, G. A. Potassium nutrition of deciduous and small fruits. In: MUNSON, R. D. Potassiun in agriculture, American Society of Agronomy for USA, 1985, p.1087-1104.

DOLINSKI, M.A.; SERRAT, B.M.; MOTTA, A.C.V.; CUQUEL, F.L.; SOUZA, S.R.; MAY-DE-MIO, L.L.; MONTEIRO, L.B. Produção, teor foliar e qualidade de frutos do pessegueiro 'Chimarrita' em função da adubação nitrogenada, na região da Lapa-PR, Revista Brasileira de Fruticultura, JaboticabalSP, v.27, n.2, ago 2005, p.295-299.

FACHINELLO, J. C.; HERTER, F. G. Diretrizes para a Produção Integrada de Frutas de Caroço, Embrapa Clima Temperado, Pelotas-RS, Circular técnico n.19, abr 2000, 46p.

GOMES, F.R.C.; FACHINELLO, J.C.; MEDEIROS, A.R.M.; GIACOBBO, C.L.; SANTOS, I.P. Influência do manejo do solo e da intensidade de raleio de frutas, no crescimento e qualidade de pêssegos, cvs. Cerrito e Chimarrita, Revista Brasileira de Fruticultura, Jaboticabal-SP, v.27, n.1, p.6063, abr 2005.

MAGNANI, M.; FREIRE, C.J.S.; MORAES, E.C. Manejo de NPK em pessegueiro (Prunus persica (L.) Batsch, In: VII Congresso Brasileiro de Fruticultura, EMPASC, SBF, Anais... Florianópolis-SC, v.4, p.1.105-1.123, 1984.

MARSCHNER, H. Mineral Nutrition of Higher Plants, 2 ed., Academic Press Limited, San Diego-CA, 1995, 889p.

MATTOS, M.L.T.; FREIRE, C.J.S.; MAGNANI, M. Produção do pessegueiro cv. Diamante, sob diferentes doses de nitrogênio aplicado ao solo, Pesquisa Agropecuária Brasileira, Brasília, v.26, n.1, p.113-117, jan. 1991.

MENGEL, K.; KIRKBY, E. A. Principles of Plant Nutrition, 4.ed., International Potash Institute, Switzerland, 1987, 687p.

MOTTA, C.V.M.; SERRAT, B.M.; FAVARETTO, N. FERTILIDADE DO SOLO. IN: MONTEIRO, L.B.; MAY-DE MIO, L.L.; SERRAT, B.M.; MOTTAA.C.; CUQUEL F. L. Fruteiras de caroço: uma visão ecológica, Curitiba, UFPR, 2004, p.49-57.

NOSAL, K., Poniedzialek, W., Kropp, K. and Porebski, S. Effectiveness of nitrogen and potassium fertilization of apple trees. Acta Hort. (ISHS) 274:361-364, 1990. 
SEAB/DERAL - Secretaria da Agricultura e do Abastecimento do Paraná - Disponível em <www.pr.gov.br/seab/deral/ efpr.xls> Acesso em: 02 ago 2006.

TAGLIAVINI, M.; ROMBOLÀ, A.D.; QUARTIERI, M.; MALAGUTI, D.; MAZZANTI, F.; MILLARD, P.; MARANGONI, B. Mineral nutrition partitioning to fruits of deciduous trees. Acta Hort. (ISHS) 512, p.131-140, 2000.

TUTIDA, I. Influência do nitrogênio e do potássio na intensidade de doenças fúngicas e na qualidade dos frutos em ameixeira, 2006, 110p. Dissertação de mestrado em agronomia na área de produção vegetal - Universidade Federal do Paraná, 2006.

VITANOVA, I.M. Determination of needs for fertilizers of plum trees. Acta Hort. (ISHS) 274, p.501-508, 1990. 